\title{
Effects of puerarin combined with conventional therapy on ischemic stroke
}

\author{
MIN YUAN ${ }^{1}$, GUIJIANG LIU ${ }^{2}$, XIUQI ZHENG ${ }^{2}$, PEI LI $^{1}$, JINGHUA LIU $^{1}$, SUJIE WANG ${ }^{1}$ and YIBIN CAO ${ }^{1}$ \\ ${ }^{1}$ Department of Neurology, Tangshan Gongren Hospital, Tangshan, Hebei 063000; \\ ${ }^{2}$ Department of Medical, Tangshan Kaiping Hospital, Tangshan, Hebei 063021, P.R. China
}

Received March 31, 2017; Accepted August 2, 2017

DOI: $10.3892 /$ etm.2017.4922

\begin{abstract}
This study evaluated the use of conventional therapy combined with puerarin on ischemic stroke. Eighty patients with ischemic stroke admitted to Tangshan Gongren Hospital from March 2014 to September 2015 were randomly divided into two groups. The control group was treated with conventional therapy. The observation group was treated with an additional puerarin injection of $400 \mathrm{mg} / \mathrm{day}$. The patients in both groups were examined for clinical neurological signs and symptoms. The levels of biochemical markers as well as changes in hemorheology were measured after the intervention and compared among the groups. Our results indicate that the language expression was significantly better in the observation group after the intervention $(\mathrm{p}<0.05)$. Additionally, the aphasia quotient was higher $(\mathrm{p}<0.05)$, the neurological deficit score was lower $(\mathrm{p}<0.05)$, and the functional ability score was higher $(\mathrm{p}<0.05)$. The plasma S-100B and NSE levels were lower $(\mathrm{p}<0.05)$, the whole blood viscosity and plasma viscosity were lower $(\mathrm{p}<0.05)$, and the levels of corticotropin-releasing hormone, corticotropin, cortisol, adrenaline and norepinephrine were lower $(\mathrm{p}<0.05)$. Our findings led to the conclusion that the use of puerarin can effectively improve the language function, reduce neurological damage, reduce blood viscosity, reduce stress response and improve quality of life.
\end{abstract}

\section{Introduction}

The stress associated with improvements in living standards in China have led to increases in the incidence of ischemic stroke (1). Ischemic stroke accounts for approximately $30 \%$ of the neurological diseases, affecting mostly middle-aged males (2). The disease course is complex and its treatment with early vascular recanalization to reduce the time of brain

Correspondence to: Dr Yibin Cao, Department of Neurology, Tangshan Gongren Hospital, 27 Wenhua Road, Lubei, Tangshan, Hebei 063000, P.R. China

E-mail: sdocwk@163.com

Key words: puerarin, ischemic stroke, neurological function, quality of life, stress response tissue ischemia and hypoxia is key to improve the prognosis of patients (3). Fast acting drugs that can expand the blood vessels, promote revascularization and therefore improve brain perfusion have most commonly been used (4). However, prominent side effects such as orthostatic hypotension, dizziness and gastrointestinal discomfort are severe (5).

Recently, a combination of Chinese and Western medical treatments has become the first choice for the treatment of ischemic stroke in China. Injectable Chinese medicine drugs are a new type of medication developed under the guidance of traditional Chinese medicine theory and modern pharmacology, and they have been used successfully against ischemic stroke (6). The main active ingredient of puerarin is an isoflavone monomer extracted from Pueraria thomsonii Benth and Pueraria lobata (Willd.) Ohwi, which has significant effects such as an antioxidant (7), stabilizing vascular endothelial cell function (8), improving microcirculation and lowering blood pressure. The safety profile and efficacy in the treatment of heart and cerebrovascular diseases have been clinically confirmed (9-11).

The present study was designed to evaluate the clinical use of conventional therapy combined with puerarin for the treatment of ischemic stroke.

\section{Materials and methods}

General information. Eighty patients admitted to Tangshan Gongren Hospital with ischemic stroke from March 2014 to September 2015 were selected. The diagnoses were carried out according to the 2010 Chinese Medical Association Ischemic Stroke Diagnosis and Treatment Standards, and confirmed by head computed tomography (CT) or magnetic resonance imaging (MRI). Patients presenting with intracranial tumors, clear intracranial arteriovenous malformations, severe cardiopulmonary, liver or kidney dysfunction before the onset, mental disorders, illiteracy, drug allergies or those not willing to take part in the study were excluded. The Ethics Committee of Tangshan Gongren Hospital approved the study and all the patients signed informed consent forms. The random number method was used to divide the patients into two groups, each of 40 cases. There were no significant differences between the groups on sex, age, course of disease, quality of life score, language expression, aphasia quotient (AQ), neurological function, plasma S-100B, NSE, hemorheology indicators, 
and levels of corticotropin-releasing hormone, corticotropin, cortisol, adrenaline or norepinephrine prior to treatment $(\mathrm{p}>0.05)$ (Table I).

Methods. The patients in the control group underwent conventional treatment of ischemic stroke, including administration of common drugs to improve brain microcirculation, expanding cerebral blood vessels to improve cerebral blood volume, timely thrombolytic therapy, interventional revascularization and anticoagulation. The patients in the observation group were treated with the same conventional therapy but with an additional daily injection of $400 \mathrm{mg}$ puerarin (batch no. 1601135, SFDA approval no. H20057386; Qingdao Jinfeng Pharmaceutical Co., Ltd., Shandong, China), adding 5\% glucose injection to make $500 \mathrm{ml}$ intravenous infusion, once a day. The duration of treatment for all the patients before assessments was one month, during which the patients were hospitalized.

Observation indicators. Language expression, AQ, neurological function and functional ability changes before and after the intervention were compared between the two groups. Levels of plasma S-100B protein, NSE, corticotropin-releasing hormone, corticotropin, cortisol, adrenaline, norepinephrine and hemorheological indicators were measured prior and subsequent to the intervention. Blood samples for the patients were collected after $8 \mathrm{~h}$ fasting periods from the elbow vein.

Evaluation standards. Language expression was assessed using the Chinese aphasia examination scale. Oral expression, listening, language understanding, reading and writing functions were scored for each patient, with possible scores ranging from 0 to 200 points (a higher the score meant a better language expression). For AQ determinations a score from 98.4 to 99.6 was considered as normal, and one below 93.8 was considered as predicitive of language impairment. Neurological function deficit was assessed using the neurological deficit score, The total score ranged from 0 to 45 points, and the higher the score, the more severe the deficit. Functional abilities were assessed using the Barthel index; the total score ranged from 0 to 100 points, with lower scores indicating life ability impairments.

Plasma S-100B and NSE levels were measured by double-antibody sandwich ELISA. The normal reference value of plasma S-100B protein was $<0.5 \mu \mathrm{g} / \mathrm{l}$, and the normal reference value of NSE was $\leq 12.5 \mu \mathrm{g} / 1$. Hemorheological indicators included blood viscosity $(\eta \mathrm{b})$, plasma viscosity $(\eta p)$ and hematocrit (HCT), the $\eta \mathrm{b}$ and $\eta p$ were measured by the capillary viscosity method. The normal reference value of $\eta \mathrm{b}$ was shear rate $115 \mathrm{~s}^{-1}$ with $1.62-1.70 \mathrm{mPa} \cdot \mathrm{sec}$ (males) and $1.63-1.71 \mathrm{mPa} \cdot \mathrm{sec}$ (females). The $\eta p$ normal reference value was shear rate $115 \mathrm{~s}^{-1}$, with $22.9 \pm 0.086 \mathrm{mPa} \cdot \mathrm{sec}$. The HCT was determined by the micro-capillary method, the normal reference value for males was $0.42-0.49$ and $0.37-0.43 \%$ for females.

Hormone levels were calculated using ELISA kits purchased from Jingmei Biotech Co., Ltd. (Shanghai, China). The normal reference value for the adrenocorticotropic hormone was $26 \mathrm{pmol} / \mathrm{l}$ (standard measurement time at 8:00 a.m.). The normal value of corticotropin-releasing hormone was
Table I. General condition.

\begin{tabular}{lrrrr}
\hline Variables & $\begin{array}{c}\text { Observation } \\
\text { group }\end{array}$ & $\begin{array}{c}\text { Control } \\
\text { group }\end{array}$ & t or $\chi^{2}$ & P-value \\
\hline Sex (male/female) & $26 / 14$ & $25 / 15$ & 0.000 & 1.000 \\
Age (years) & $48.5 \pm 2.1$ & $48.6 \pm 2.1$ & 0.213 & 0.832 \\
$\begin{array}{l}\text { Course of disease } \\
\text { (months) }\end{array}$ & $3.2 \pm 0.3$ & $3.3 \pm 0.3$ & 1.491 & 0.140 \\
$\begin{array}{l}\text { Quality of life } \\
\text { score }\end{array}$ & $81.5 \pm 2.3$ & $81.6 \pm 2.3$ & 0.194 & 0.846 \\
\hline
\end{tabular}

Table II. Comparison of language skills before and after intervention (mean \pm standard deviation).

\begin{tabular}{lcccc}
\hline Groups & Before & After & t-test & P-value \\
\hline Observation & $61.9 \pm 5.5$ & $163.1 \pm 21.0$ & 29.484 & $<0.001$ \\
Control & $62.1 \pm 5.6$ & $111.2 \pm 16.9$ & 17.442 & $<0.001$ \\
t-test & 0.161 & 12.177 & - & - \\
P-value & 0.872 & $<0.001$ & - & - \\
\hline
\end{tabular}

$160-210 \mathrm{ng} / \mathrm{ml}$. The normal value of serum cortisol was 80-550 nmol/1 (standard measurement time at 8:00 a.m.). The normal value for adrenaline was $<480 \mathrm{pmol} / 1$. Finally, the normal value of norepinephrine was $615-3240 \mathrm{pmol} / \mathrm{l}$.

Statistical analysis. The SPSS 13.0 statistical software (IBM SPSS, Chicago, IL, USA) was used for statistical analysis. The measurement data were expressed as mean \pm standard deviation (mean $\pm \mathrm{SD}$ ). The comparison of the mean values between the two groups was performed by t-test. A comparison between the two groups was made using a $\chi^{2}$ test. $\mathrm{P}<0.05$ was considered to indicate a statistically significant difference.

\section{Results}

Comparison of language expression ability before and after intervention. There was no significant difference in the average language expression ability of the two groups before intervention $(\mathrm{p}>0.05)$. However, after the intervention, the language expression ability in the observation group was significantly improved compared to that in the control group before and after the intervention $(\mathrm{p}<0.05)$ (Table II).

$A Q$ before and after intervention. There was no significant difference in the AQ between the two groups before the intervention $(p>0.05)$. After the intervention, the average AQ of the observation group was significantly higher than that of the control group before and after the intervention $(\mathrm{p}<0.05)$ (Table III).

Comparison of neurological deficit and functional ability after intervention. After the intervention, the scores of neurological deficits in the observation group were significantly lower than those in the control group $(\mathrm{p}<0.05)$. The average score of 
Table III. AQ before and after intervention (mean \pm standard deviation).

\begin{tabular}{lcccc}
\hline Groups & Before & After & t-test & P-value \\
\hline Observation & $45.6 \pm 2.8$ & $86.5 \pm 6.2$ & 38.024 & $<0.001$ \\
Control & $46.1 \pm 2.9$ & $73.1 \pm 3.5$ & 37.569 & $<0.001$ \\
t-test & 0.784 & 11.903 & - & - \\
P-value & 0.435 & $<0.001$ & - & - \\
\hline
\end{tabular}

$\mathrm{AQ}$, aphasia quotient.

Table IV. Comparison of neurological function scores and living ability scores after intervention (mean $\pm \mathrm{SD}$ ).

\begin{tabular}{lcc}
\hline Groups & $\begin{array}{c}\text { Neurological } \\
\text { function score }\end{array}$ & $\begin{array}{c}\text { Living ability } \\
\text { score }\end{array}$ \\
\hline Observation & $13.6 \pm 2.0$ & $79.1 \pm 5.2$ \\
Control & $23.5 \pm 3.1$ & $51.0 \pm 4.5$ \\
t-test & 16.972 & 25.851 \\
P-value & $<0.001$ & $<0.001$ \\
\hline
\end{tabular}

Table V. Comparison of plasma S-100B protein and NSE levels after intervention (mean \pm standard deviation).

\begin{tabular}{lcc}
\hline Groups & NSE $(\mathrm{ng} / \mathrm{ml})$ & S-100B protein $(\mathrm{ng} / \mathrm{ml})$ \\
\hline Observation & $2.1 \pm 0.2$ & $0.6 \pm 0.1$ \\
Control & $5.6 \pm 1.0$ & $1.5 \pm 0.2$ \\
t-test & 21.706 & 25.456 \\
P-value & $<0.001$ & $<0.001$ \\
\hline
\end{tabular}

Table VI. Hemorheology results after intervention (mean \pm standard deviation).

\begin{tabular}{lccc}
\hline Groups & $\eta \mathrm{b}(\mathrm{mPa} \cdot \mathrm{sec})$ & $\eta \mathrm{p}(\mathrm{mPa} \cdot \mathrm{sec})$ & $\mathrm{HCT}(\%)$ \\
\hline Observation & $1.63 \pm 0.05$ & $20.32 \pm 0.03$ & $40.21 \pm 2.03$ \\
Control & $2.11 \pm 0.06$ & $25.61 \pm 0.08$ & $40.31 \pm 2.10$ \\
t-test & 38.869 & 499.689 & 0.217 \\
P-value & $<0.001$ & $<0.001$ & 0.829
\end{tabular}

HCT, hematocrit.

functional ability in the observation group was significantly better than that in the control group $(\mathrm{p}<0.05)$ (Table IV).

Comparison of plasma $S-100 B$ protein and NSE levels after intervention. The levels of plasma S-100B protein and NSE in the observation group were significantly lower than those in the control group $(\mathrm{p}<0.05)$ (Table $\mathrm{V})$.
Table VII. Levels of corticotropin-releasing hormone, corticotropin and cortisol in the two groups after intervention (mean \pm standard deviation).

\begin{tabular}{lccc}
\hline Groups & $\begin{array}{c}\text { Corticotropin-releasing } \\
\text { hormone }(\mathrm{ng} / \mathrm{ml})\end{array}$ & $\begin{array}{c}\text { ACTH } \\
(\mathrm{pmol} / \mathrm{l})\end{array}$ & $\begin{array}{c}\text { Cortisol } \\
(\mathrm{nmol} / \mathrm{l})\end{array}$ \\
\hline Observation & $311.3 \pm 31.3$ & $46.8 \pm 8.1$ & $658.9 \pm 33.1$ \\
Control & $525.3 \pm 52.6$ & $105.6 \pm 13.3$ & $1715 \pm 113.2$ \\
t-test & 22.112 & 23.881 & 56.666 \\
P-value & $<0.001$ & $<0.001$ & $<0.001$
\end{tabular}

Table VIII. Adrenaline and norepinephrine levels (pmol/1, mean \pm standard deviation).

\begin{tabular}{lcc}
\hline Groups & Adrenaline & Norepinephrine \\
\hline Observation & $51.1 \pm 2.3$ & $121.3 \pm 6.5$ \\
Control & $89.5 \pm 6.1$ & $256.3 \pm 21.3$ \\
t-test & 37.253 & 38.340 \\
P-value & $<0.001$ & $<0.001$ \\
\hline
\end{tabular}

Hemorheology results after intervention. There was no significant difference in the HCT between the two groups after the intervention, and $\eta \mathrm{b}$ and $\eta p$ in the observation group were lower than those in the control group $(\mathrm{p}<0.05)$ (Table VI).

Levels of corticotropin-releasing hormone, corticotropin and cortisol in the two groups after intervention. The levels of corticotropin-releasing hormone, corticotropin and cortisol in the observation group were significantly lower than those in the control group after treatment $(\mathrm{p}<0.05)$ (Table VII).

Adrenaline and norepinephrine levels. After treatment, the levels of adrenaline and norepinephrine in the observation group were significantly lower than those in the control group $(\mathrm{p}<0.05)($ Table VIII).

\section{Discussion}

Ischemic stroke has a very acute onset, and if not treated immediately, it often leads to severe disability or death. This disease occurs commonly in elderly patients, in whose morbidity and mortality rates rank first among neurological diseases (12). Ischemic stroke is mainly due to cerebral arterial atherosclerosis. Vascular endothelial cell damage causes lipid plaques to attach to the vessel walls, causing vascular stenosis and luminal blockage. The result is retarded cerebral blood flow and increased blood viscosity, leading to thrombosis and eventually causing ischemic stroke (13). Puerarin is the active ingredient of the traditional Chinese medicine puerarin. The clinical preparation of puerarin injection contains isoflavone compounds, which can dilate blood vessels and improve the microcirculatory function (14). The injection has been widely used in clinical treatment of vascular-related diseases, and the clinical effects have been confirmed. 
The puerarin injection treatment in the observation group seemed to provide a beneficial effect against ischemic stroke. In comparison to the results in the control group, the language expression ability and AQ of the patients in the observation group were significantly improved. This suggests puerarin can effectively improve the language ability after the onset of the disease and reduce the incidence and severity of language dysfunction. Puerarin can effectively cross the blood-brain barrier, therefore it probably is effective by dilating the cerebral blood vessels, reducing cerebral vascular resistance, improving cerebral blood flow and oxygen supply, and thereby reducing the impact of ischemia and hypoxia on the language centers, while at the same time, promoting cerebral angiogenesis, and reducing the apoptosis of brain neurons after hypoxia (15). Furthermore, our study showed the neurological deficit was less severe and the living capacity of patients was improved in the patients of the observation group, while plasma S-100B protein and NSE levels were significantly higher than those in the control group. It is possible that the isoflavone in puerarin works directly in the cerebrovascular smooth muscle by reducing calcium influx to improve cerebrovascular endothelial cell function (16). The isoflavone can also activate the body plasmin system, reducing fibrinogen levels and improving the blood supply in the distal cerebral infarction area in ischemic stroke, competitively antagonizing excitatory amino acid neurotoxicity (17). It is probably as a combination of these functions that puerarin allows the neuron cells of patients to get better blood supply and recover function faster, and as a consequence the quality of life is improved and there is a decrease in the neurological alteration of biochemical markers.

When comparing the hemorheology results after intervention, we found that the $\eta \mathrm{b}$ and $\eta p$ in the observation group were significantly lower than the same factors in the control group. We believe puerarin effectively reduced blood viscosity. It has been suggested that puerarin inhibits the thrombin-induced release of serotonin in platelets, thereby reducing platelet aggregation, adjusting the balance between blood coagulation and fibrinolysis $(18,19)$, and that it has a certain effect on the clearance of oxygen-free radicals in the microcirculation (20).

Finally, our results on the levels of corticotropin-releasing hormone, corticotropin and cortisol showed the levels in the observation group were significantly lower than those in the control group, and the levels of adrenaline and norepinephrine were also significantly lower. The use of puerarin is known to reduce adrenaline related hormone secretion levels and the stress response. A study in the literature has shown the use of puerarin in combination with other conventional therapies reduces blood viscosity, promoting cerebral blood flow, reducing red blood cell aggregation, reducing secondary cerebral thrombosis, and improving cellular respiration throughout the body (21).

We conclude that adding puerarin daily injections to the conventional therapy against ischemic stroke can effectively improve the language function, reduce neurological damage, reduce blood viscosity, reduce stress response and improve the quality of life of patients.

\section{References}

1. Zheng QH, Li XL, Mei ZG, Xiong L, Mei QX, Wang JF, Tan LJ, Yang SB and Feng ZT: Efficacy and safety of puerarin injection in curing acute ischemic stroke: A meta-analysis of randomized controlled trials. Medicine (Baltimore) 96: e5803, 2017.
2. Li P, Bai J, Dong B, Lu Y, Zhang S, Guo S, Tan N, Zhao M, Du $S$ and Cao P: In vivo pharmacokinetics of puerarin via different drug administration routes based on middle cerebral artery occlusion model. Eur J Drug Metab Pharmacokinet 7: 719-727, 2017.

3. Wu C, Zhao L, Rong Y, Zhu G, Liang S and Wang S: The pharmacokinetic screening of multiple components of the Nao Mai Tong formula in rat plasma by liquid chromatography tandem mass spectrometry combined with pattern recognition method and its application to comparative pharmacokinetics. J Pharm Biomed Anal 131: 345-354, 2016.

4. Xue Q, Liu Y, He R, Yang S, Tong J, Li X, Chen Y and Xu X: Lyophilized powder of catalpol and puerarin protects neurovascular unit from stroke. Int J Biol Sci 12: 367-380, 2016.

5. Zhang X, Liu XT and Kang DY: Traditional Chinese patent medicine for acute ischemic stroke: An overview of systematic reviews based on the GRADE approach. Medicine (Baltimore) 95: e2986, 2016. doi: 10.1097/MD.0000000000002986.

6. Liu B, Tan Y, Wang D and Liu M: Puerarin for ischaemic stroke. Cochrane Database Syst Rev 2: CD004955, 2016.

7. Liu X, Mei Z, Qian J, Zeng Y and Wang M: Puerarin partly counteracts the inflammatory response after cerebral ischemia/reperfusion via activating the cholinergic anti-inflammatory pathway. Neural Regen Res 8: 3203-3215, 2013.

8. Wu M, Liang S, Ma L, Han Y, Zhang X and Xu C: Effects of delayed puerarin treatment in long-term neurological outcomes of focal ischemic stroke in rats. Indian J Pharmacol 46: 157-160, 2014.

9. Liu Y, Xue Q, Li X, Zhang J, Fu Z, Feng B, Chen Y and Xu X: Amelioration of stroke-induced neurological deficiency by lyophilized powder of catapol and puerarin. Int J Biol Sci 10: 448-456, 2014.

10. Wu XD, Wang C, Zhang ZY, Fu Y, Liu FY and Liu XH: Puerarin attenuates cerebral damage by improving cerebral microcirculation in spontaneously hypertensive rats. Evid Based Complement Alternat Med 2014: 408501, 2014.

11. Wang N, Zhang Y, Wu L, Wang Y, Cao Y, He L, Li X and Zhao J: Puerarin protected the brain from cerebral ischemia injury via astrocyte apoptosis inhibition. Neuropharmacology 79: 282-289, 2014

12. Ouyang $\mathbf{Z}$, Zhao $\mathbf{M}$, Tang $\mathbf{J}$ and Pan L: In vivo pharmacokinetic comparisons of ferulic acid and puerarin after oral administration of monomer, medicinal substance aqueous extract and Nao-De-Sheng to rats. Pharmacogn Mag 8: 256-262, 2012.

13. Lim DW, Lee C, Kim IH and Kim YT: Anti-inflammatory effects of total isoflavones from Pueraria lobata on cerebral ischemia in rats. Molecules 18: 10404-10412, 2013.

14. Zhao LX, Liu AC, Yu SW, Wang ZX, Lin XQ, Zhai GX and Zhang QZ: The permeability of puerarin loaded poly(butylcyanoacrylate) nanoparticles coated with polysorbate 80 on the blood-brain barrier and its protective effect against cerebral ischemia/reperfusion injury. Biol Pharm Bull 36: 1263-1270, 2013.

15. Chang Y, Hsieh CY, Peng ZA, Yen TL, Hsiao G, Chou DS, Chen CM and Sheu JR: Neuroprotective mechanisms of puerarin in middle cerebral artery occlusion-induced brain infarction in rats. J Biomed Sci 16: 9-11, 2009.

16. Wan $\mathrm{H}$, Zhu $\mathrm{H}$, Tian $\mathrm{M}, \mathrm{Hu} \mathrm{X}$, Yang J, Zhao $\mathrm{C}$ and Zhang $\mathrm{H}$ : Protective effect of chuanxiongzine-puerarin in a rat model of transient middle cerebral artery occlusion-induced focal cerebral ischemia. Nucl Med Commun 29: 1113-1122, 2008.

17. Tan Y, Liu M and Wu B: Puerarin for acute ischaemic stroke. Cochrane Database Syst Rev 23: CD004955, 2008.

18. Wu B, Liu M, Liu H, Li W, Tan S, Zhang S and Fang Y: Meta-analysis of traditional Chinese patent medicine for ischemic stroke. Stroke 38: 1973-1979, 2007.

19. Li R, Song J, Wu W, Wu X and Su M: Puerarin exerts the protective effect against chemical induced dysmetabolism in rats. Gene 595: 168-174, 2016.

20. Jiang M, Yun Q, Niu G, Gao Y, Shi F and Yu S: Puerarin prevents inflammation and apoptosis in the neurocytes of a murine Parkinson's disease model. Genet Mol Res 15: 112-115, 2016.

21. Zhao L, Wang Y, Liu J, Wang K, Guo X, Ji B, Wu W and Zhou F: Correction to protective effects of genistein and puerarin against chronic alcohol-induced liver injury in mice via antioxidant, anti-inflammatory, and anti-apoptotic mechanisms. J Agric Food Chem 64: 8463-8470, 2016. 